\title{
On the origin of field-aligned beams at the quasi-perpendicular bow shock: multi-spacecraft observations by Cluster
}

\author{
H. Kucharek ${ }^{1}$, E. Möbius ${ }^{1}$, M. Scholer ${ }^{2}$, C. Mouikis ${ }^{1}$, L. M. Kistler ${ }^{1}$, T. Horbury ${ }^{3}$, A. Balogh ${ }^{3}$, H. Réme ${ }^{4}$, and \\ J. M. Bosqued ${ }^{4}$ \\ ${ }^{1}$ Department of Physics and Institute for the Study of Earth, Oceans and Space (EOS) University of New Hampshire, \\ Durham, NH 03824, USA \\ ${ }^{2}$ MPI für extraterrestrische Physik, Garching, Germany \\ ${ }^{3}$ Imperial College, London, UK \\ ${ }^{4}$ CESR, Toulouse, France
}

Received: 26 September 2003 - Revised: 6 April 2004 - Accepted: 5 May 2004 - Published: 14 July 2004

Part of Special Issue "Spatio-temporal analysis and multipoint measurements in space"

\begin{abstract}
Two distinct populations of reflected and accelerated ions are known to originate from quasi-perpendicular shocks, gyrating ions and reflected ion beams. Recent observations under such bow shock conditions with Cluster have shown strong evidence that both particle distributions appear to emerge from the same reflection process. In this paper the basic production mechanism of field-aligned beams has been investigated by using CLUSTER multi-spacecraft measurements. We have analyzed several quasi-perpendicular shocks with the Cluster Ion Spectrometry experiment (CIS) and followed the spatial and temporal evolution of the reflected and transmitted ion populations across the shock. These observations show that the field-aligned beams most likely result from effective scattering in pitch angle during reflection in the shock ramp. Investigating a low Mach number shock, leakage of a fraction of the thermalized ion distribution in the downstream region does not appear to be the source as the volume in phase space occupied by beam ions is empty downstream of the shock ramp.
\end{abstract}

Key words. Interplanetary physics (planetary bow shocks) Space plasma physics (shock waves) - Radio science (magnetosphere physics)

\section{Introduction}

A very prominent and well-known feature of the Earth's bow shock is the presence of ions backstreaming into the foreshock region (e.g. Lin et al., 1974). The properties of these upstream ions have been intensively studied in the past by in situ spacecraft observations. It was soon found that there are two basically different spatially separated populations of

Correspondence to: $\mathrm{H}$. Kucharek

(kucharek@atlas.sr.unh.edu) backstreaming ions (e.g. Gosling et al., 1978). Reflected ions form collimated ion beams with energy of a few $\mathrm{keV}$ along the interplanetary magnetic field from the bow shock (e.g. Paschmann et al., 1980; Bonifazi and Moreno, 1981; Thomsen et al., 1983a, b; Schwartz et al., 1983). These beams primarily emanate from regions of the bow shock where the shock normal angle $\Theta_{B n}$ is between $70^{\circ}$ and $45^{\circ}$, that is, the quasi-perpendicular regime. In the quasi-parallel regime of the Earth's bow shock upstream ions exhibits a more broader, isotropic distribution and relatively flat energy spectrum extending to well beyond $100 \mathrm{keV}$ (e.g. Ipavich et al., 1981; Scholer et al., 1981). In the transition region between these two regions the ion population is called "intermediate ions" (Paschmann et al., 1979). The source of these ions, and their basic acceleration mechanisms have been under intensive observational and theoretical investigation for the last forty years but, they are still not completely understood.

There have been a number of proposals to produce the field-aligned ion beams. Sonnerup (1969) demonstrated that solar wind protons could easily be energized if the bow shock could manage to turn them around in such a way that they left the shock reasonably well field-aligned. It was assumed that the particle energy was preserved in the de Hoffmann-Teller frame, where the flow is field-aligned and the interplanetary electric field is null. Paschmann et al. (1980) actually found that the peak energy of the ion beams as a function of the magnetic field orientation relative to the solar wind and to the shock normal agrees well with the prediction of Sonnerup's model. Edmiston et al. (1982) proposed leakage of the heated downstream plasma as a mechanism for upstream field-aligned beams. They concluded, however, that fractional densities up to $1 \%$ could only escape upstream for $\Theta_{B n}$ between $40^{\circ}$ and $55^{\circ}$. Tanaka et al. (1983) presented a more sophisticated leakage model by taking into account the non-Maxwellian particle distribution downstream: part of the 

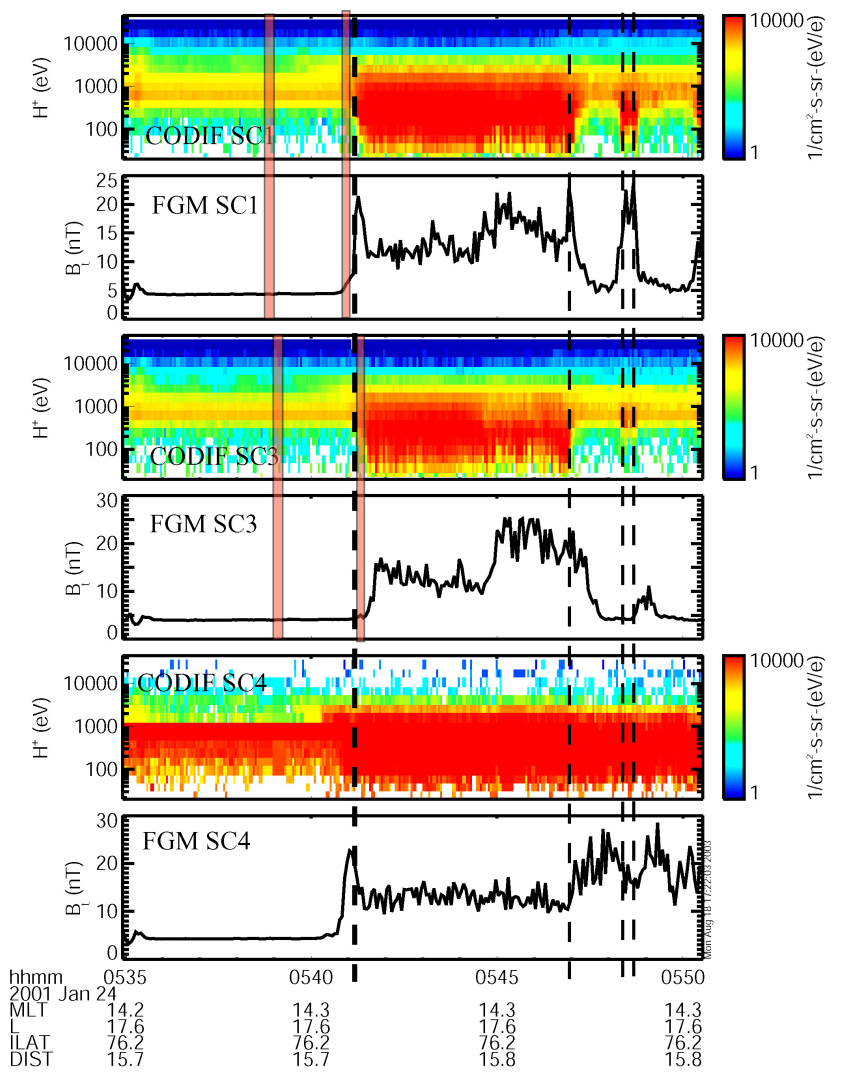

Fig. 1. From top to bottom: Colored spectrogram of the $\mathrm{H}^{+}$differential particle flux and the magnetic field magnitude B from S/C 1, S/C 3, and S/C4, for the time period 05:30-05:50 UT on 24 January 2001. The vertical lines indicate the bow shock crossings of S/C 3 .

solar wind ions incident on a quasi-perpendicular shock are specularly reflected, accelerated by the upstream $\mathbf{v} \times \mathbf{B}$ motional electric field to $\sim 2 \mathrm{v}_{1}$, where $\mathrm{v}_{1}$ is the upstream flow speed. These ions are transmitted downstream and constitute after sufficient pitch angle scattering an approximately isotropic high energy shell in the downstream rest frame. Part of the shell may escape upstream as a field-aligned beam. Since isotropization is not assumed to be immediate behind the shock, suprathermal particles downstream of the perpendicular shock can exit the shock, where the shock, due to its curvature, has a lower value of $\Theta_{B n}$. Burgess and Luhmann (1986) have tested one part of the scenario proposed by Tanaka et al. (1983). They studied the propagation of low energy (but suprathermal) protons through the magnetosheath in a model obtained from gas-dynamic simulations. According to their results, leakage is a function of $\Theta_{B n}$, and local leakage could be important for shock normal angles larger than $\sim 60^{\circ}$.

Burgess (1987) used one-dimensional hybrid simulations to study direct reflection of protons from a shock and the subsequent formation of field-aligned beams. Since it turned out that the protons in the beams originate from the wings of the incident Maxwellian distribution, he used a test particle technique, combined with the hybrid simulation, to enhance the particle statistics in this part of phase space. He found that the density of the reflected ions decreases with increasing $\Theta_{B n}$, as well as with increasing Mach number. As $\Theta_{B n}$ increases direct reflection is more and more from the wing, suggesting that non-Maxwellian distributions with some high energy tail could well produce high intensity field-aligned beams above $\sim 60^{\circ}$.

In this paper we will analyze specularly reflected ions, field-aligned ion beams, and downstream thermalization during various quasi-perpendicular shock crossings by CLUSTER, in order to gain new insight into the formation mechanism of field-aligned ion beams. In particular, we will analyze a high Mach number, high beta shock encounter that shows a very strong ion beam and immediate thermalization. This investigation is followed by a detailed analysis of a low Mach number, low beta shock crossing which shows a persisting anisotropy in the downstream velocity distribution. Finally, we will investigate a multi-spacecraft measurement of a high Mach number, low beta bow shock encounter of the CLUSTER spacecraft. On the basis of these observations and of the observations reported earlier by Möbius et al. (2001) we will test the models and we will propose a generalized model for the ion beam formation at quasiperpendicular shocks.

\section{Observations and instrumentation}

For this investigation we used data from the fluxgate magnetometer FGM (Balogh et al., 1997) and the Cluster Ion Spectrometry (CIS) on board the CLUSTER II spacecraft. The CIS unit consists of two complementary sensors. The Composition and Distribution Function (CODIF) analyzer provides the 3-D velocity distribution function of the major ion species $\left(\mathrm{H}^{+}, \mathrm{He}^{2+}, \mathrm{He}^{+}\right.$and $\left.\mathrm{O}^{+}\right)$in the energy range from spacecraft potential to $40 \mathrm{keV} / \mathrm{e}$ with a time resolution up to one spin period. The Hot Ion Analyzer (HIA) provides the 3$D$ velocity distribution with high energy and angle resolution, but without species discrimination. Both sensors are based on a top hat electrostatic analyzer design, which provides a $360^{\circ}$ instantaneous field-of-view, divided into angular pixels. A detailed description of the instruments may be found in Réme et al. (1997) and Möbius et al. (1998). During the operational phase of the CLUSTER mission, the spacecraft encountered a huge number of bow shock crossings under a variety of different plasma conditions. We begin our investigation of the source and the formation mechanism with a high beta, quasi-perpendicular shock crossing, with a high Mach number.

\subsection{High Mach number shock}

Figure 1 shows a shock crossing at 24 January 2001, which is also discussed in a paper by Möbius et al. (2001) among the early results of the CLUSTER mission. From top to bottom, we show the energy spectrum and the magnetic field magnitude for three spacecraft. Due to a failure in the power 
supply the energy spectrum for spacecraft 2 is missing. At around 05:40 UT the Cluster spacecraft encounter the perpendicular bow shock. The shock normal angle $\Theta_{B n}$ at this time has been determined by minimum variance analysis to be $70^{\circ}$, the Alfvénic Mach number $\mathrm{M}_{A}=11$ and the plasma beta $\beta=0.36$.

It should be noted here that all determined shock normal angles in this paper have been verified by the spacecraft timing method and by model bow shock (Slavin and Holzer, 1981). The results agreed within an error of \pm 5 . For more detailed information, we would like to refer to Horbury et al. (2002).

At this shock ion beams parallel to the magnetic field and reflecting gyrating gyrophase-bunched ions were observed simultaneously on all spacecrafts (see below). Only during the time period 05:35-05:40 UT were all spacecraft continuously and almost simultaneously in the solar wind. This can be seen from the narrow maximum of the proton flux at around $1 \mathrm{keV}$. At around 05:41 UT the spacecraft enter the bow shock, indicated by a sharp increase in the magnetic field and a broadening of the $\mathrm{H}^{+}$energy distributions. Note that SC4 is operating in high gain mode and therefore partly saturated. After 05:42 UT all spacecraft cross the bow shock in the sequence SC 4, SC 1, and finally SC 3. At 05:47 UT SC 1 and 3 exit again from the magnetosheath in the reverse sequence and encounter the bow shock. All shock crossings are marked with a dashed line. For our study we have examined the first shock crossing at 05:41 UT in more detail. The red vertical bars indicate time periods at which the spacecraft have been upstream of the bow shock in the solar wind and at the shock ramp. Figure 2 shows the proton distributions' functions in the $\mathrm{B}-\mathrm{V}_{s w}$ plane, as measured by the CODIF instrument on board CLUSTER spacecraft number 1 (top panel) and 3 (lower panel) during the passage through the Earth's bow shock at these times. The particle distribution in velocity space parallel and perpendicular to the interplanetary magnetic field $\mathbf{B}$ (indicated by an arrow) is shown in the plane that also includes the solar wind, as the spacecraft passes from upstream (left hand panels) through the shock ramp (right hand panels) to downstream. The distribution of gyrating ion, the beams, as well as the solar wind are clearly visible. Upstream of the Earth's bow shock both spacecraft measure the solar wind distribution in this representation at large parallel, but small perpendicular velocities. A beam of reflected ions with intensity several orders of magnitude lower than the intensity of the solar wind ions can be seen, which propagates anti-parallel to the magnetic field. Inside the ramp both spacecraft also observe a distribution of the gyrating ions, in addition to the beam and solar wind ions. As can be seen the beam and the gyrating ion distribution are connected to each other and form a combined distribution. This is a strong indication that their origin is in fact the same; or, in other words, the ion beam emerges from the combined gyrating distribution. Immediately after passing the shock ramp the distribution is fully thermalized, that is, the distribution is equally spread out in parallel and perpendicular velocity (see Sect. 2.2). During the crossing of this

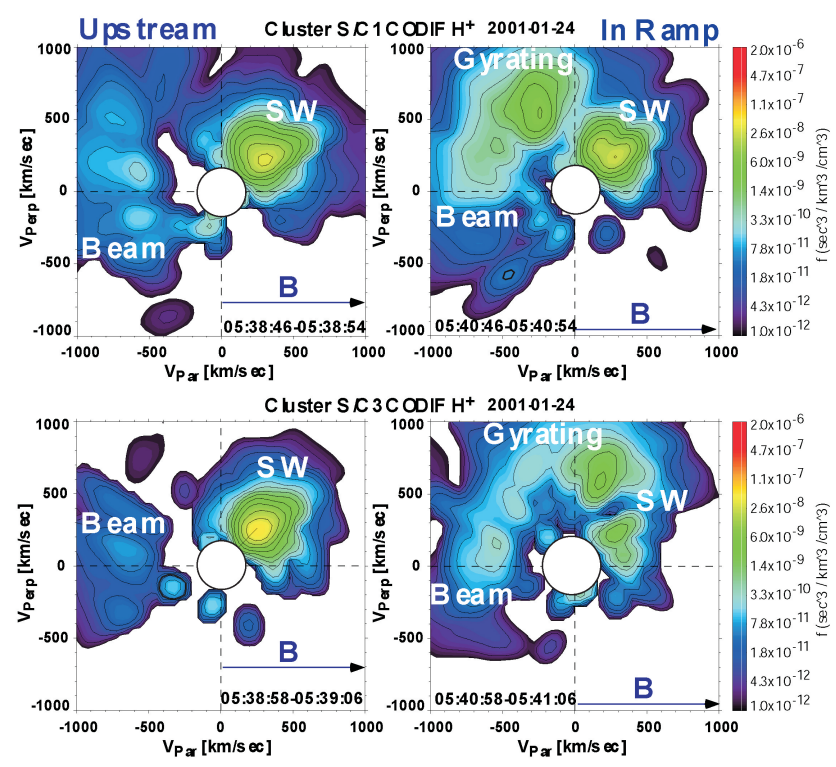

Fig. 2. Color-coded velocity distributions in $\mathrm{B}-\mathrm{V}_{s w}$ plane (B defines the direction of $\mathrm{v}_{\text {para }}$ ) for SC1 and SC3 for the bow shock crossing at 24 January 2001. Also indicated are the magnetic field orientation, the solar wind, the gyrating ions and the ion beam.

strong shock, the scale length for thermalization is very short, which is due to strong wave-particle scattering in the turbulent downstream wave field. In principle, any mechanism which produces particles at the shock front will lead to beamlike distributions, because ions will stream predominantly along magnetic field lines (Burgess et al., 1987). However, at this point two models seem to be able to explain the production of the reflected beam at this quasi-perpendicular shock.

1. Reflection in the ramp into gyrating ions and immediate scattering by Alfvén waves can provide the source of these beams. Ions with small pitch angles can, according to Scholer et al. (2000), escape along B. The beam strength would then depend on $\mathrm{M}_{A}$ and plasma $\beta$.

2. Also, thermalization in the downstream region and leakage upstream along B can explain the observations, as the fully thermalized distribution may be the source, where $\Theta_{B n}$ is small enough so that $\mathrm{v} \|$ exceeds the convection velocity relative to $B$. According to Tanaka et al. (1983), the ions would be able to escape upstream across the bow shock.

In the following we will extend our study to low Mach number shocks, which are known to produce a downstream distribution with a high perpendicular to parallel anisotropy (Sckopke et al., 1990), in order to see whether ion beams emerge from the downstream region or from the ramp.

2.2 Low Mach number vs. high Mach number: transmission and thermalization

On 31 March 2001, the CLUSTER spacecraft encountered a series of low beta and low Mach number shocks. We have 


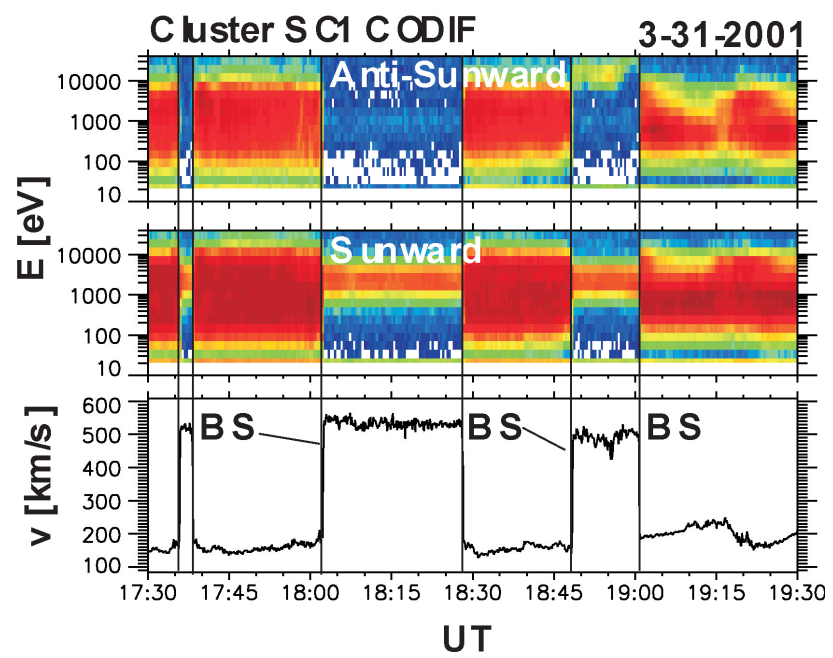

Fig. 3. $\mathrm{H}^{+}$spectrograms taken anti-sunward and in the sunward direction and solar wind speed for a series of low Mach number bow shock crossing on 31 March 2001.

chosen these shock crossings as our first examples of low Mach number shocks. Figure 3 shows part of this series of shock crossings. The energy spectra in anti-sunward (top panel) and sunward directions (middle panel) and the bulk velocity (lower panel) for spacecraft 1 are shown. We concentrate on $\mathrm{SC} 1$ data because the CODIF instrument was in a high time resolution mode. For these shock crossings (indicated by full vertical lines), a $\Theta_{B n}$ of $74.5^{\circ}, \mathrm{M}_{A}=3, \beta=0.03$ has been determined. Between the last two shock crossings energetic ions have been observed with a strong antisunward anisotropy (see top panel of Fig. 3 at 18:02 UT). In Fig. 4 we compare the distributions observed upstream and downstream of the high Mach number shock, discussed in Sect. 2.1, with the corresponding distributions obtained during the crossing of a low Mach number shock on 31 March 2001. The top half of this figure shows the total magnetic field (top panel) and the two distributions for the high Mach number shock (24 January 2001, 05:40 UT), as seen by SC1. The shaded bars indicate the time period during which the distributions are taken. In the lower half of this figure we show the distributions and the magnetic field magnitude for the low Mach number crossing on 31 March at 18:02 UT, as measured on SC1. Again, the time periods at which the distributions are integrated are marked with shaded bars. At the shock ramp we find the solar wind and the gyrating ion distribution. In the case of the high Mach number shock the distribution behind the shock ramp is immediately thermalized and completely isotropic, whereas in the low Mach number shock a large perpendicular to parallel anisotropy remains in accordance with the results by Sckopke et al. (1990). This is most probably due to less pitch angle scattering in the shock ramp and in the downstream region of the low Mach number shock as compared to the high Mach number shock.

The two models (leakage versus scattering in the shock ramp) differ in where the ion beams are produced. If the

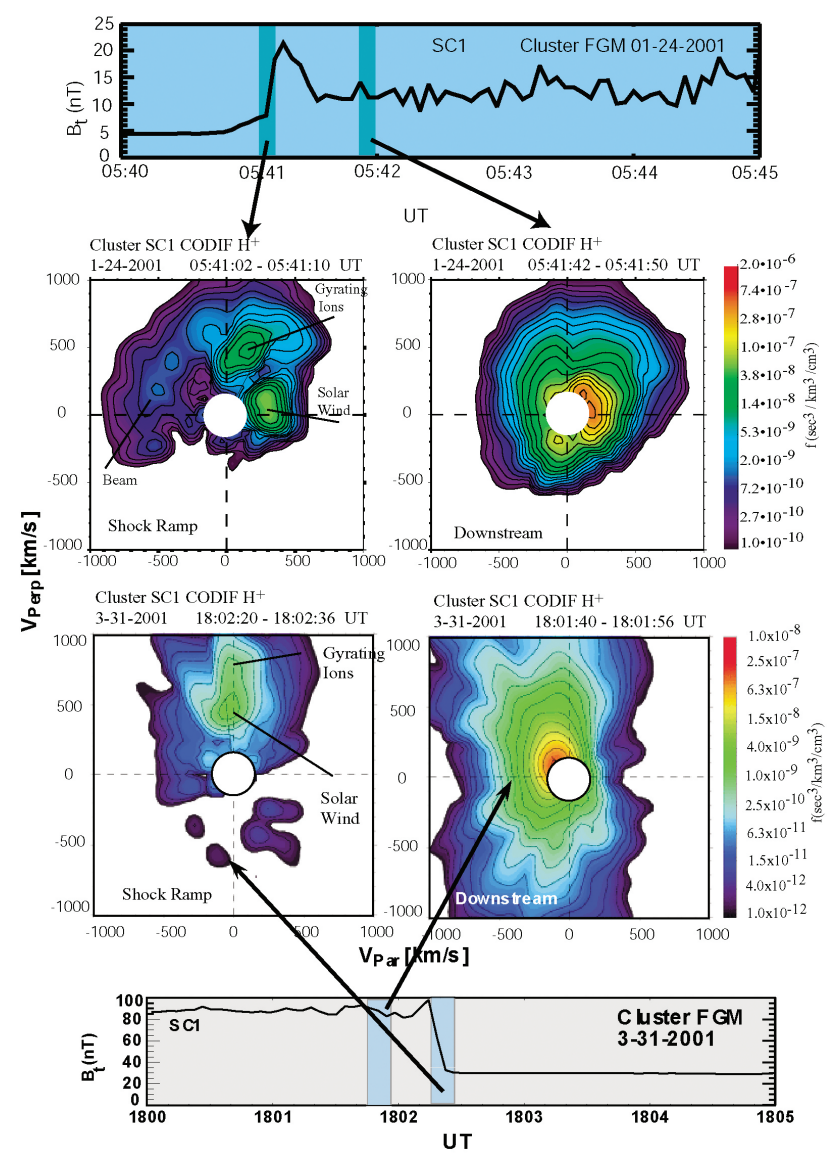

Fig. 4. Color-coded velocity distribution in the $\mathrm{V}_{\perp}-\mathrm{V}_{\|}$plane in the shock ramp and downstream of the Earth's bow shock for 31 March and for 24 January 2001. The observation intervals are indicated by the shaded bar in the corresponding panel of the magnetic field.

source population of the field-aligned beams is located downstream and leakage from downstream produces these beams, an ion population should then be present downstream. At the high Mach number shock, where we see a very rapid thermalization downstream, there might be a possibility for a contribution, or even significant contribution, from downstream leakage to the field-aligned beam distribution. However, this model apparently does not provide ions in the phase space portion from where ions could leak upstream at the low Mach number shock, where thermalization is slow and a remaining temperature anisotropy persists downstream. Yet between 18:48 UT and 19:02 UT we find a substantial energetic ion population which shows strong anisotropy.

Investigating a case with a strong perpendicular to parallel anisotropy in the downstream distribution, such a case should make it possible to discriminate between the two models. Therefore, we followed the temporal evolution of the ion distribution measured by CIS/CODIF during the passage of SC1 from downstream to upstream at 18:48 UT on 31 March 2001. Figure 5 shows a composite plot during this crossing, including a snapshot at the shock ramp. The top panel shows the magnetic field, and in the lower panel 


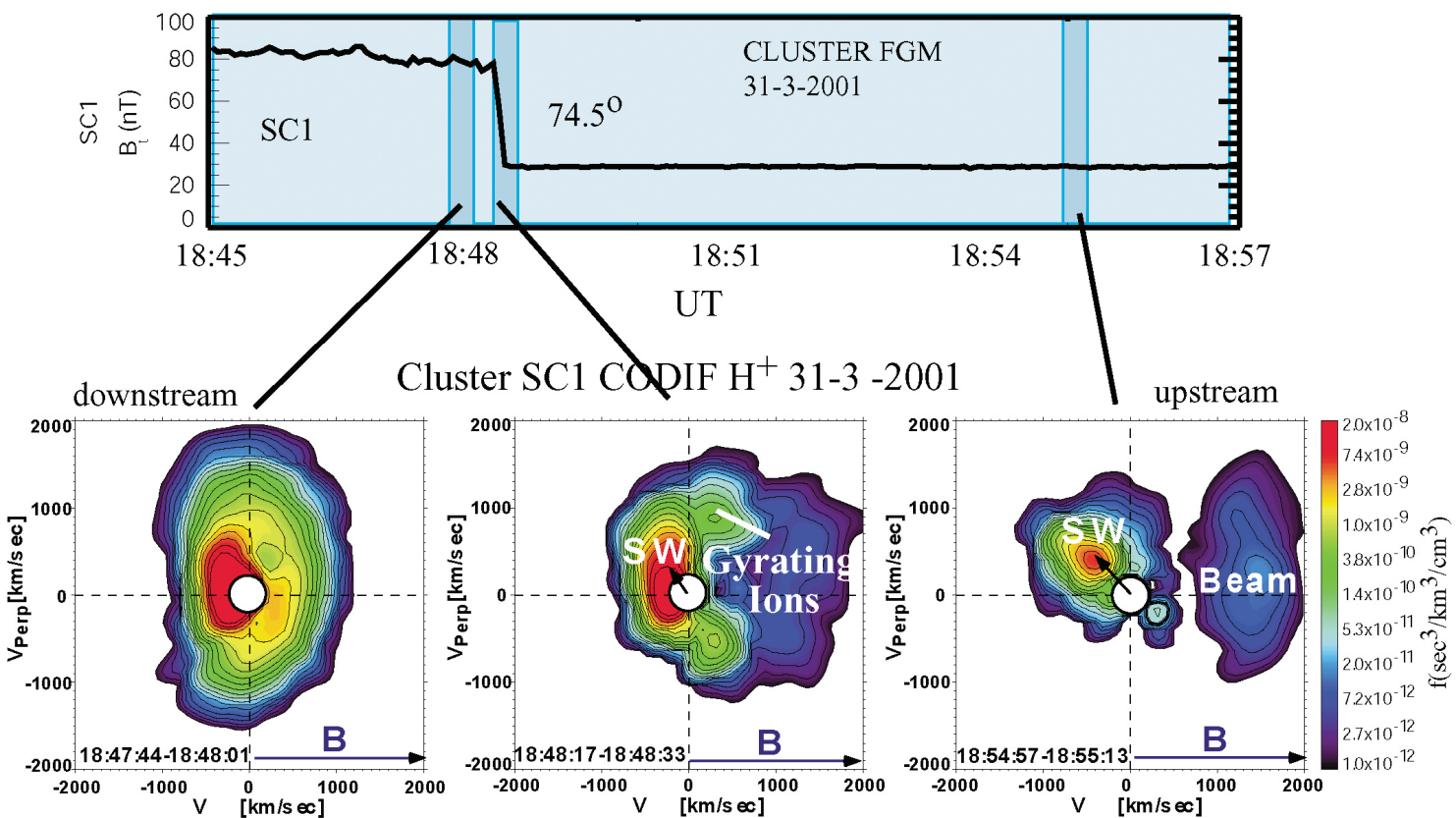

Fig. 5. Magnetic field (top panel) and ion distribution (lower panel) in velocity space downstream, in the ramp, and upstream of the bow shock.

the ion distributions parallel and perpendicular to the interplanetary magnetic field (the mean magnetic field orientation is indicated by arrows) are shown for three different locations: downstream, at the ramp, and upstream of the bow shock. The dark blue shaded areas in the plot of the magnetic field profile indicate the integration time for the ion distributions. Downstream, the shape of the ion distribution is more elongated in the perpendicular than in the parallel direction. The phase space is filled with ions up to a parallel velocity of $1000 \mathrm{~km} / \mathrm{s}$. In the shock ramp gyrating ions appear, while phase space density extends towards parallel velocity exceeding substantially the limit of $\approx 1000 \mathrm{~km} / \mathrm{s}$. Upstream of the bow shock (see right hand distribution) this part of the distribution decouples from the core distribution and forms a collimated beam along the mean interplanetary magnetic field. It should be noted that the beams occupy a portion of the phase space that is empty downstream.

This observation is a strong indication that the ion beam is produced inside the shock ramp by intense wave particle scattering rather than leaking from far downstream. The measured ion beam flux is of the order of $2 \%$ of the incoming solar wind for the high Mach number shock and a factor of 50 lower for the low Mach number shock (not shown in this paper).

However, this is a single spacecraft measurement that provides a time sequence with snapshots of the distributions, which may be changing in time. Phase space density may increase or decrease, and the situation shown above could be coincidental. Thus, single spacecraft measurements cannot provide conclusive evidence.
2.3 Multi-spacecraft observations: a key to the basic beam production mechanism

Unlike single spacecraft that only provide single point measurements along the spacecraft trajectory, a multi-spacecraft mission such as CLUSTER provides simultaneous readings at different locations. Such a measurement from the CLUSTER spacecraft taken on 2 January 2002, at around 14:45 UT, is shown in Fig. 6. At this time the spacecraft cross a high Mach number shock $\left(\mathrm{M}_{A}=5\right)$ with a shock normal angle of $72^{\circ}$. The top and the bottom part of this figure show the distributions in $\mathrm{v} \perp$ vs. $\mathrm{v} \|$ space for $\mathrm{SC} 1$ and $\mathrm{SC} 4$, respectively. The middle part of this figure shows the energy spectra (top panel) and the magnetic field (lower panel) of three different spacecraft SC1, SC3, and SC4. The vertical lines in the spectrograms indicate the time periods in which the distributions have been taken. As the figure shows, spacecraft 1 is located close to the bow shock, because it crosses the shock first. $\mathrm{SC} 3$ and SC4 are in a more distant location. They enter the downstream region later and leave it earlier, almost simultaneously. Therefore, only SC1 is able to measure ion distributions in the distant downstream region as seen at 14:42 UT. At this position the downstream distribution is still not fully isotropic: it is more elongated in the perpendicular direction than in the parallel direction where the phase space appears empty around $\mathrm{V}_{\|}=-1000 \mathrm{~km} / \mathrm{s}$ (vertical line). Later in time, at 14:45:00 UT, SC1 is still downstream whereas SC4 is already at the shock ramp. Ions are accelerated up to parallel velocities of $-1000 \mathrm{~km} / \mathrm{s}$ and beyond. At 14:47:00 UT SC1 is in the shock ramp whereas SC4 is already upstream of the shock. The distribution observed by SC4 shows the solar wind and a field-aligned ion beam. The distribution observed 

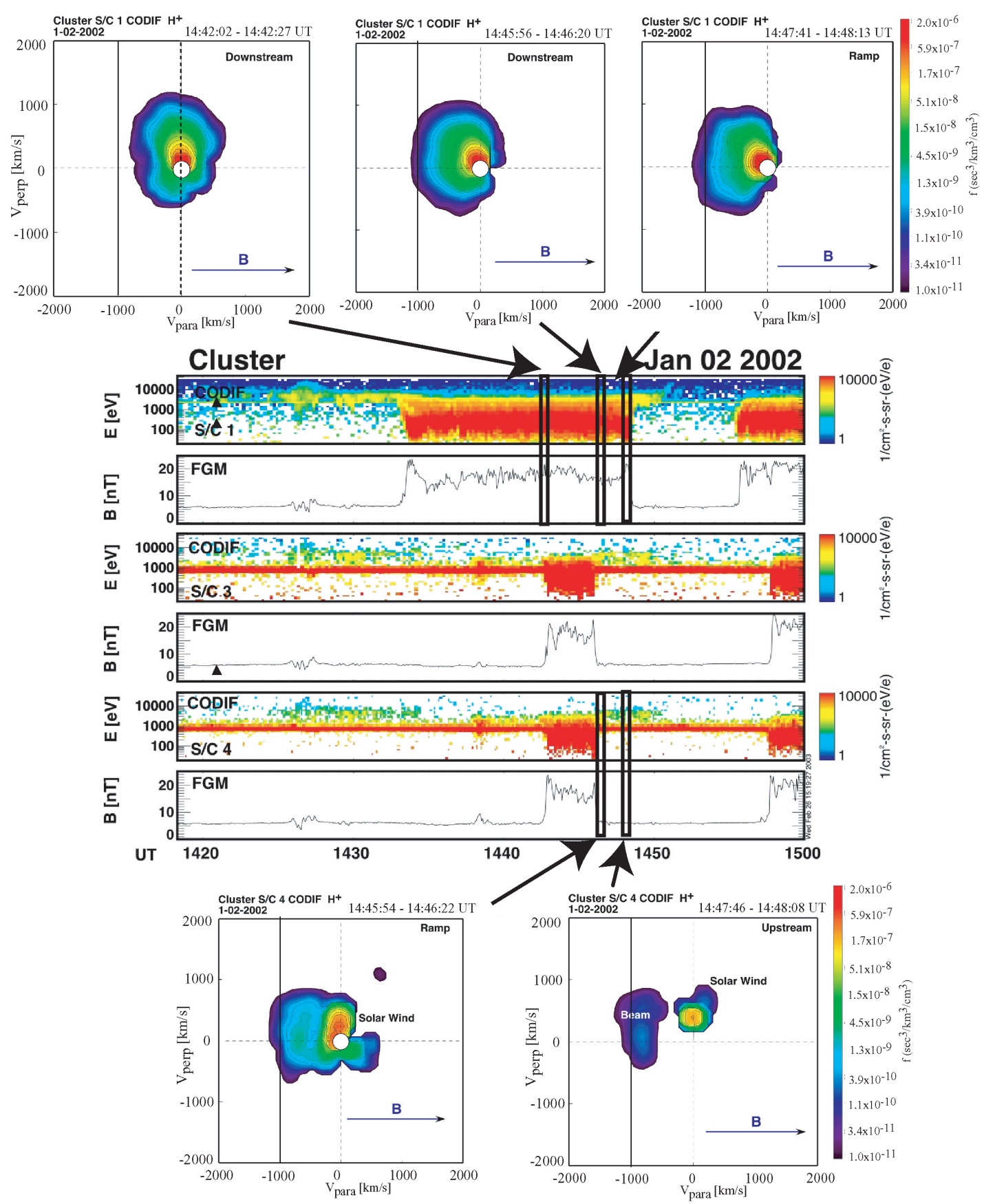

Fig. 6. Multi-spacecraft observations during crossing of the Earth's bow shock on 2 January 2003. The top and lower panels show the colorcoded velocity distribution in the $\mathrm{V}_{\perp}-\mathrm{V}_{\|}$plane during a bow shock crossing. The middle part shows the differential flux and the magnetic field of the different spacecraft.

by $\mathrm{SC} 1$ at the same time has expanded to higher parallel velocities, but it is different from the distribution measured by SC4 in the shock ramp. This is most probably due to the fact that the distributions have been taken at different locations in the ramp. However, following the temporal/spatial development of the ion distribution it becomes evident that the ion beam mostly originates from the shock ramp and not from downstream because there are no ions detected in the phase space that is associated with the upstream ion beam.
How can these ions escape upstream? An escaping particle has to rely on its guiding center motion, which has to carry it away from the shock along the field line against the convection of the magnetic field of the solar wind bulk flow.

To simplify the discussion the processes at the shock are often transformed into the Hoffmann-Teller (HT) frame, thus eliminating the motional electric field. Assuming that the HT frame is the natural frame for reflection, the velocity of the field-aligned ions can be calculated from $\theta_{B n}, \theta_{V n}$, 
$\theta_{B V}$, and the velocity of the solar wind (see Schwartz and Burgess, 1983). Furthermore, assuming that direct reflection of solar wind ions by the shock conserves energy, the velocity of the field-aligned beam $\mathrm{V}_{b}$ is of the order of the component of the solar wind parallel to the magnetic field $\mathrm{V}_{s w \|}$, or $\left(\mathrm{V}_{b} / \mathrm{V}_{s w \|}=1\right)$. If adiabatic leakage of heated magnetosheath ions would be the main mechanism, the ratio of the beam velocity versus solar wind velocity would be of the $\mathrm{V}_{b} / \mathrm{V}_{s w \|}=\cos \Theta_{B n}$ (see Schwartz et al., 1983). Using the conditions for the shock crossing on 31 March 2001, at 18:47 UT $\left(\theta_{B n}=74.5^{\circ}, \theta_{V n}=7^{\circ}, \theta_{B V}=86.2^{\circ}, \mathrm{V}_{s w}=545 \mathrm{~km} / \mathrm{s}\right.$, $\mathrm{V}_{b}=1500 \mathrm{~km} / \mathrm{s}$ one calculates a ratio $\mathrm{V}_{b} / \mathrm{V}_{s w \|}=-0.3$. This would indicate that the ion beam propagates downstream in the HT frame and should not be seen upstream. If one assumes an ideal planar shock, a critical angle of $\theta_{B n}=67^{\circ}$ would be needed to obtain $\mathrm{V}_{b} / \mathrm{V}_{s w \|}=0$ that the particles can escape upstream in the HT frame. This angle is somewhat smaller than the measured shock normal angle. It appears as if the basic escape condition is violated and the conditions are far from reflection under conservation of energy $\left(\mathrm{V}_{b} / \mathrm{V}_{s w \|}=1\right)$. However, it is well known that perpendicular shocks are dynamic and small-scale structures can lead to a deviation of the average $\theta_{B n}$. During the crossing on 31 March that is associated with the ion beam the Earth's bow shock is moving with a velocity of about $30 \mathrm{~km} / \mathrm{s}$ towards the Earth. During the preceding crossing, which is not associated with an ion beam, the shock is moving away from the Earth (not shown here). This reflects that the bow shock is not stationary and the local $\theta_{B n}$ may be variable, both modifying the critical conditions so that ions can escape upstream. At the escape, conditions for the ions are most probably marginally fulfilled for the crossing at 18:47 UT, whereas at 18:30 UT they are not.

While we have concluded from the sequence of observations of ion distributions that leakage from far downstream cannot be the main process for the formation of the ion beam, the observations at these large $\theta_{B n}$ suggest that direct reflection of solar wind ions at the bow shock is most likely also not the basic formation process of the field-aligned ion beams.

\section{Summary and conclusion}

The goals of this study were to investigate the thermalization properties of the quasi-perpendicular shock and the basic production mechanism of the field-aligned ion beams. We have investigated shock crossings of a high and several low Mach number shocks, with multi-spacecraft measurements. In particular, we have investigated the spatial and temporal evolution of the proton distribution during these bow shock crossings. The results of this investigation can be summarized as follows: gyrating and beam ions originate from the same distribution. Compared to low Mach number shocks, high Mach number shocks are more efficient in thermalizing of the incoming plasma. It produces an intensive fieldaligned ion beam (up to $2 \%$ of the incoming solar wind inten- sity during high Mach number conditions). Low Mach number shocks tend to be less efficient to thermalize the incoming plasma. The ion distributions of low Mach number shocks show a large temperature anisotropy and their ion beams are less intense $(\approx 50$ times less than the intensity of a strong shock in the case of 24 January 2001). In both cases the field-aligned ion beam appears to be produced in the shock ramp and does not leak from far downstream.

Processes right in the shock ramp and the dynamic of the shock itself produce these ion beams. A detailed analysis of the shock structure and shock potential is therefore important and it will provide new insights into the reflection and acceleration properties of perpendicular shocks. Therefore, scattering in the shock ramp seems to be a major process that produces these ion beams. A detailed analysis on shock crossings where the escape conditions for ions are marginally fulfilled might provide a deeper insight into the formation processes for field-aligned beams at high shock normal angles. However, this can only be done in a statistical study, which is beyond the scope of this paper.

We therefore conclude the presented multi-spacecraft observations support the following, more generalized formation mechanism of field-aligned beams at the quasi-perpendicular Earth's bow shock: Part of the incoming solar wind is reflected and accelerated at the shock to form a gyrating ion distribution. Pitch angle scattering in the shock ramp will produce a small fraction of the gyrating ions, which have a high velocity parallel to the magnetic field. All ions out of this scattered distribution which have a velocity component parallel to the shock normal larger than the convection speed of interplanetary magnetic field at the shock ramp will escape upstream of the shock and form a field-aligned ion beam. The intensity of the ion beam upstream will then be determined by the pitch angle scattering in the shock ramp and not primarily on the shock geometry. The fluxes of ion beams show significant differences between the spacecraft and vary with time, which seems to reflect spatial and temporal upstream structures. This leaves a number of questions still unanswered. What determines the fraction of reflected ions that forms the gyration ion distribution? What fraction of these ions will escape upstream? What are the critical shock parameters that govern the ion beam formation? How important is internal structure of the shock and what is the impact of the shock potential on particle reflection and acceleration? Future observational studies of reflected and the transmitted ion distributions, combined with numerical simulation, are necessary to improve our knowledge of the downstream thermalization, ion injection and acceleration at the Earth's bow shock.

Acknowledgements. This work has been supported by NASA contracts: NAG5-10131 and NAG5-11804.

Topical Editor T. Pulkkinen thanks R. P. Lin and another referee for their help in evaluating this paper. 


\section{References}

Balogh, A., Dunlop, M. W., Cowley, S., Southwood, D., Thomlinson, J. G., Glassmeier, K. H., Musmann, G., Lühr, H., Buchert, S., Acuita, M., Fairfield, D. H., Slavin, J. A., Riedler, W., Schwingenschuh, K., Kivelson, M. G., and the CLUSTER magnetometer team: The Cluster magnetic field investigation, Space Sci. Rev., 79, 65, 1997.

Bonifazi, C. and Moreno, G.: Reflected and diffuse ions backstreaming from the earth's bow shock, 1. Basic properties, J. Geophys. Res., 86, 4397, 1981.

Burgess, D.: Simulations of backstreaming ion beams formed at oblique shocks by direct reflection, Ann. Geophys., 5, 133-145, 1987.

Burgess, D. and Luhmann, J. G.: Scatter-free propagation of low energy protons in the magnetosheath: implications for the production of field-aligned beams by non-thermal leakage, J. Geophys. Res., 91, 1439-1449, 1986.

Edmiston, J. P., Kennel, C. F., and Eichler, D.: Escape of heated ions upstream of quasi-parallel shocks, Geophys. Res. Lett., 9, 531-534, 1982

Gosling, J. T., Asbridge, J. R., Bame, S. J., Paschmann, G., and Sckopke, N.: Observations of two distinct populations of the bow shock ions in the upstream solar wind, Geophys. Res. Lett., 5, 957, 1978

Horbury, T. S., Cargill, P. J., Lucek, E. A., Eastwood, J., Balogh, A., Dunlop, M. W., Fornacon, K.-H., and Georgescu, E.: Four spacecraft measurements of the quasiperpendicular terrestrial bow shock: Orientation and motion, J. Geophys. Res., 107 (A8), 1208, doi:10.1029/2001JA000273, 2002.

Ipavich, F. M., Galvin, A. B., Gloeckler, G., Scholer, M., and Hovestadt, D.: A statistical survey of ions observed upstream of the earth's bow shock - Energy spectra, composition, and spatial variation, J. Geophys. Res., 86, 4337-4342, 1981.

Lin, R. P., Meng, C.-I., and Anderson, K. A.: 30- to 100-keV protons upstream from the earth's bow shock, J. Geophys. Res., 79, 489, 1974.

Möbius, E., Kistler, L. M., Popecki, M., Crocker, K., Granoff, M., Jiang, Y., Sartori, E., Ye, V., Rème, H., Sauvaud, J. A., Cros, A., Aoustin, C., Camus, T., Médale, J. L., Rouzaud, J., Carlson, C. W., McFadden, J. P., Curtis, D. W., Heetderks, H., Croyle, J., Ingraham, C., Shelley, E. G., Klumpar, D., Hertzberg, E., Klecker, B., Ertl, M., Eberl, F., Kästle, H., Künneth, E., Laeverenz, P., Seidenschwang, E., Parks, G. K., McCarthy, M., Korth, A., Gräwe, B., Balsiger, H., Schwab, U., and Steinacher, M.: The 3-D Plasma Distribution Function Analyzers With Time-ofFlight Mass Discrimination for CLUSTER, FAST and Equator$\mathrm{S}$, Measurement Techniques in Space Plasmas, edited by Pfaff, R., Borowski, J., Young, D., Geophys. Monograph, 102, 243, 1998.

Möbius, E., Kucharek, H., Mouikis, C., Geogescu, E., Kistler, L. M., Popecki, M. A., Scholer, M., Bosqued, J. M., Réme, H., Carlson, C. W., Klecker, B., Korth, A., Parks, G. K., Sauvand, J. C., Balsiger, H., Bavassano-Cattaneu, M.-B., Dandouras, I., DiLellis, A. M., Eliasson, L., Formisano, V., Hobury, T., Lennart- son, W., Lundin, R., McCarthy, M., McFadden, J. P., and Paschmann, G.: Observation of the spatial and temporal structure of field-aligned beam and gyrating ring distributions at the quasiperpendicular bow shock with Cluster CIS, Ann. Geophys., 19, 1411, 2001.

Paschmann, G., Sckopke, N., Bame, S. J., Asbridge, J. R., Gosling, J. T., Russell, C. T., and Greenstadt, E. W.: Association of lowfrequency waves with suprathermal ions in the upstream solar wind, Geophys. Res. Lett, 6, 209-212, 1979.

Paschmann, G., Sckopke, N., Papamastorakis, I., Asbridge, J. R., Bame, S. J., and Gosling, J. T.: Energetization of solar wind ions by reflection from the earth's bow shock, J. Geophys. Res., 85, 4689, 1980.

Paschmann, G., Sckopke, N., Bame, S. J., and Gosling, J. T.: Observations of gyrating ions in the foot of the nearly perpendicular bow shock, Geophys. Res. Lett., 9, 881, 1982.

Réme, H., Bosqued, J. M., Sauvaud, J. A., Cros, A., Dandouras, J., Aoustin, C., Martz, Ch., Médale, J. L., Rouzaud, J., Möbius, E., Crocker, K., Granoff, M., Kistler, L. M., Hovestadt, D., Klecker, B., Paschmann, G., Ertl, M., Künneth, E., Carlson, C. W., Curtis, D. W., Lin, R. P., McFadden, J. P., Croyle, J., Formisano, V., DiLellis, M., Bruno, R., Bavassano-Cattaneo, M. B., Baldetti, B., Chionchio, G., Shelley, E. G., Ghielmetti, A. G., Lennartson, W., Korth, A., Rosenbauer, H., Szemerey, I., Lundin, R., Olson, S., Parks, G. K., McCarthy, M., and Balsiger, H.: The CLUSTER Ion Spectrometry Experiment, Space Sci. Rev., 79, 303, 1997.

Scholer, M., Ipavich, F. M., and Gloeckler, G.: Beams of protons and alpha particles greater than approximately $30 \mathrm{keV} / \mathrm{charge}$ from the earth's bow shock, J. Geophys. Res., 86, 4374-4378, 1981.

Scholer, M., Kucharek, H., and Giacalone, J.: Cross-field diffusion of charged particles and the problem of ion injection and acceleration at quasi-perpendicular shocks J. Geophys. Res., 105, $18285,2000$.

Sckopke, N., Paschmann, G., Brinca, A. L., Carlson, C. W., and Lühr, H.: Ion thermalization in quasi-perpendicular shocks involving reflected ions, J. Geophys. Res., 95, 6337, 1990.

Schwartz, S. J., Thomsen, M. F., and Gosling, J. T.: Ions upstream of the earth's bow shock: A theoretical comparison of alternative source populations, J. Geophys. Res., 88, 2039-2047, 1983.

Slavin, J. A. and Holzer, R. E.: Solar wind flow about the terrestrial planets, 1, Modeling bow shock position and shape J. Geophys. Res., 86, 11 401-11418, 1981.

Sonnerup, B. U. O.: Acceleration of particles reflected at a shock front, J. Geophys. Res., 74, 1301, 1969.

Tanaka, M., Goodrich, C. C., Winske, D., and Papadopoulos, K.: A Source of the backstreaming ion beams in the foreshock region, J. Geophys. Res., 88, 3046, 1983.

Thomsen, M. F., Schwartz, S. J., and Gosling, J. T.: Observational evidence on the origin of ions upstream of the earth's bow shock, J. Geophys. Res., 88, 7843-7852, 1983a.

Thomsen, M. F., Gosling, J. T., Bame, S. J., Feldman, W. C., Paschmann, G., and Schopke, N.: Field-aligned beams upstream of the Earth's bow shock: Evidence for a magnetosheath source, Geophys. Res. Lett., 10, 1207-1210, 1983b. 